\title{
Corrigendum: Alterations of Gut Microbiome in the Patients With Severe Fever With Thrombocytopenia Syndrome
}

\footnotetext{
OPEN ACCESS

Edited by:

George Grant,

University of Aberdeen

United Kingdom

Reviewed by:

Keun Hwa Lee,

Jeju National University, South Korea

Keita Matsuno,

Hokkaido University, Japan

*Correspondence:

Lifen Hu

lifen.hu@163.com

Jiabin $\mathrm{Li}$

lijiabin@ahmu.com.cn

${ }^{\dagger}$ Co-first authors

Specialty section:

This article was submitted to

Infectious Diseases,

a section of the journal

Frontiers in Microbiology

Received: 28 November 2018

Accepted: 30 January 2019

Published: 19 February 2019

Citation:

Xu H, Wei Y, Ma H, Liu Y, Zhang Y, Hu L and Li J (2019) Corrigendum: Alterations of Gut Microbiome in the

Patients With Severe Fever With

Thrombocytopenia Syndrome.

Front. Microbiol. 10:251

doi: 10.3389/fmicb.2019.00251
}

\begin{abstract}
Honghai $X u^{1,2,3 t}$, Yuanyuan Wei ${ }^{3,4 t}$, Hongqiu Ma ${ }^{4}$, Yanyan Liu ${ }^{3}$, Yalong Zhang ${ }^{3}$, Lifen Hu ${ }^{1,3 *}$ and Jiabin $\mathrm{Li}^{1,3 *}$

${ }^{1}$ Department of Infectious Diseases, The First Affiliated Hospital of Anhui Medical University, Hefei, China, ${ }^{2}$ Department of Pathology, The First Affiliated Hospital of Anhui Medical University, Hefei, China, ${ }^{3}$ Anhui Center for Surveillance of Bacterial Resistance, Hefei, China, ${ }^{4}$ Department of Hospital Infection Control, The First Affiliated Hospital of Anhui Medical University, Hefei, China
\end{abstract}

Keywords: sever fever with thrombocytopenia syndrome (SFTS), gut microbiome, 16S rDNA sequencing, Bacteroidetes, Firmicutes, Proteobacteria, clinical symptoms, key serum enzymes

\section{A Corrigendum on}

Alterations of Gut Microbiome in the Patients With Severe Fever With Thrombocytopenia Syndrome

by Xu, H., Wei, Y., Ma, H., Liu, Y., Zhang, Y., Hu, L., et al. (2018). Front. Microbiol. 9:2315. doi: 10.3389/fmicb.2018.02315

In the original article, there was a mistake in Figure $\mathbf{2}$ as published. In this article, Figure $\mathbf{2}$ is used to introduce the taxonomic differences of gut microbiota between SFTS and healthy control groups. According to the reviewers' helpful comments, we tried to integrate one chart and three tables from the original manuscript into one new figure. However, there are some misalignments in the label of the Y-axis in Figure 2. When integrating these four images (A, B, C, and D) into Figure 2, the order of the $\mathrm{Y}$-axis annotations of each graph were reversed. The corrected Figure 2 appears below.

The authors apologize for this error and state that this does not change the scientific conclusions of the article in any way.

Conflict of Interest Statement: The authors declare that the research was conducted in the absence of any commercial or financial relationships that could be construed as a potential conflict of interest.

Copyright $\odot 2019 \mathrm{Xu}$, Wei, Ma, Liu, Zhang, Hu and Li. This is an open-access article distributed under the terms of the Creative Commons Attribution License (CC BY). The use, distribution or reproduction in other forums is permitted, provided the original author(s) and the copyright owner(s) are credited and that the original publication in this journal is cited, in accordance with accepted academic practice. No use, distribution or reproduction is permitted which does not comply with these terms. 
A

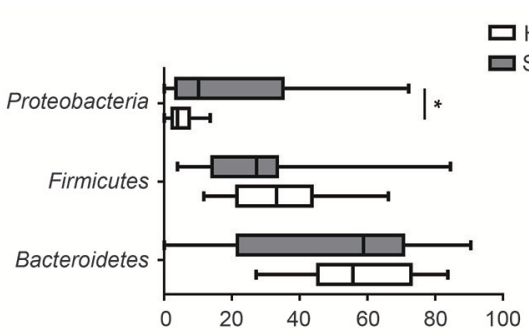

C

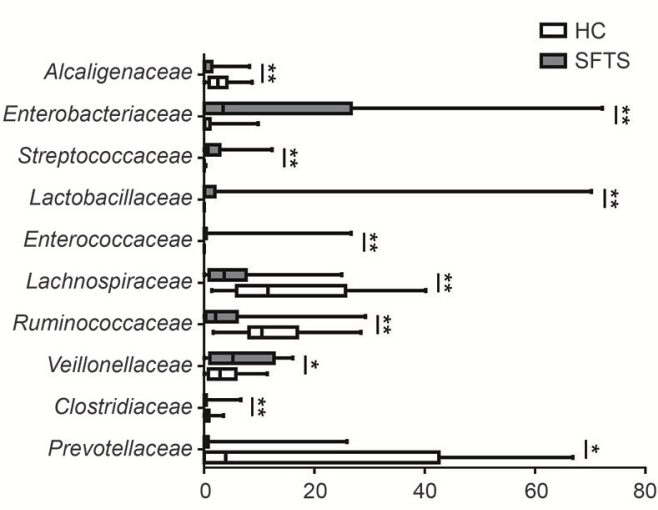

B

Betaproteobacteria $\mathbf{\mathbb { W }}$

口 $\mathrm{HC}$

口SFTS

Gammaproteobacteria

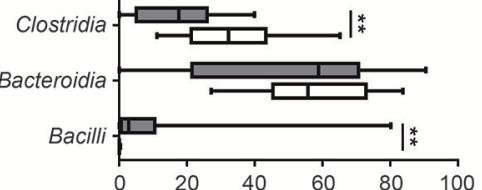

D

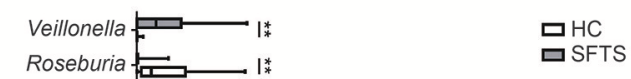

Lachnospira

Coprococcus :

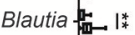

Anaerostipes - $1 *$

Faecalibacterium 旦回 |*

Ruminococcus 泗——

Streptococcus

Lactobacillus

SMB53 * * *

Escherichia

Sutterella

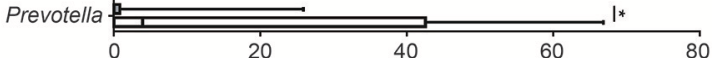

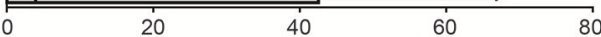

FIGURE 2 | Taxonomic differences of gut microbiota between SFTS, health control groups. Comparison of relative abundance at the bacterial phylum (A), class (B), family (C), genus (D) between the two groups. ${ }^{*} P<0.05,{ }^{* *} P<0.01$. 